\title{
CD95 ligand - death factor and costimulatory molecule?
}

\author{
O Janssen ${ }^{\star, 1}, \mathrm{~J}$ Qian $^{1}$, A Linkermann ${ }^{1}$ and D Kabelitz ${ }^{1}$ \\ 1 Institute for Immunology, Medical Center Schleswig-Holstein, Campus Kiel, \\ Michaelisstrasse 5, D-24105 Kiel, Germany \\ * Corresponding author: O Janssen. Tel: + 49-431-5973377; Fax: + 49-431- \\ 5973335; E-mail: ojanssen@email.uni-kiel.de
}

Received 24.4.03; revised 12.6.03; accepted 20.6.03; published online 1 August 2003 Edited by $\mathrm{T}$ Ferguson

\begin{abstract}
The CD95 ligand is involved as a death factor in the regulation of activation-induced cell death, establishment of immune privilege and tumor cell survival. In addition, CD95L may serve as a costimulatory molecule for T-cell activation. Alterations in expression or shedding of membrane and soluble CD95L are associated with numerous diseases, and underscore the pathophysiological relevance of the CD95/CD95L system. In most cases, the causal link between altered CD95L expression and pathophysiology is unknown. Given the potency of the molecule to regulate death and survival of many different cell types, the control of CD95L production, transport, storage, shedding and inactivation is of tremendous biological and clinical interest. This review summarizes the current knowledge, hypotheses and controversies about CD95L as a multifunctional ligand and receptor. It considers the different roles of membrane and soluble forms of CD95L and the complex networks of intracellular dynamics of protein trafficking, as well as the potential bidirectional signal transduction capacity of CD95L, with a focus on molecular interactions that have been worked out over the past years.

Cell Death and Differentiation (2003) 10, 1215-1225, doi:10.1038/ sj.cdd. 4401305

Published online 1 August 2003
\end{abstract}

Keywords: costimulation; reverse signal transduction; CD95 ligand; protein transport; secretory lysosomes; death factor; counterattack; immune privilege

\footnotetext{
Abbreviations: aa, amino acids; ADAM, a-disintegrin-and metalloproteinase; AICD, activation-induced cell death; CD95L, CD95 ligand; FBP, forming-binding protein; GST, glutathione-Stransferase; MALDI-TOF, matrix-assisted laser disorption ionization time of flight; (M)MP, (matrix)metalloproteinase; PACSIN, protein kinase $C$ and casein kinase substrate in neurons; PRD, proline-rich domain; SH, Src homology; TCR, T-cell receptor; TIL, tumor-infiltrating lymphocytes; TNF-R, tumor necrosis factor receptor
}

\section{Tissue and Cellular Expression of CD95L}

The CD95 ligand (CD95L, Apo-1L, FasL, CD178) is a 281amino-acid-containing type II transmembrane protein of the TNF family of death factors (Figure 1). ${ }^{1}$ Its death-inducing function is best documented in the context of activationinduced cell death (AICD) in T cells. ${ }^{2}$ CD95L is expressed as a death factor in cytotoxic $T$ lymphocytes (CTL) to kill virally infected or transformed target cells and in natural killer (NK) cells, where it is upregulated by CD16 engagement and cytokines including IL-2 and IL-12. ${ }^{3}$ Similarly, high levels of intracellular CD95L have been detected in monocytic cells with an inducible release upon activation. ${ }^{4}$ Under physiological conditions, CD95L is implicated in the control of erythroid differentiation, ${ }^{5}$ angiogenesis in the eye ${ }^{6}$ and skin homeostasis. ${ }^{7}$ Furthermore, CD95L expression can be induced in a variety of cell types by stress including heat shock, exposure to chemotherapeutic agents or radiation, growth factor withdrawal and viral infection. ${ }^{8}$

CD95L expression has been associated with the establishment of immune privilege and tumor survival, although this is the matter of a still unresolved controversy. ${ }^{9-11}$ In fact, constitutive or inducible membrane expression of CD95L was shown for numerous cell and tissue types including the eye, mature spermatozoa, uterus and placenta, lung, thyrocytes, keratinocytes (upregulated by UV-B light), CD68positive histiocytes in the dermis, ${ }^{12}$ neutrophils, mature erythroblasts, breast epithelial and vascular endothelial cells, astrocytes (upregulated by IL-1, IL-6, TNF- $\alpha$ and IFN- $\gamma$ ), microglia (downregulated by IFN- $\gamma$ ) and intervertebral discs. The assumption, however, that CD95L confers immune privilege and protection on organ transplants has been challenged by studies indicating a differential pro- or anti-inflammatory effect of CD95L depending on the microenvironmental context. Also, CD95L alone is not sufficient to prevent allograft rejection and other immunosuppressive factors such as TGF- $\beta$ seem to be involved.

Many of the classical transcription factors have been identified to regulate $\mathrm{CD} 95 \mathrm{~L}$ expression under different conditions (see Kavurma and Khachigian ${ }^{13}$ for review). Briefly, NFAT, NF- $k$ B, c-myc, IRF-1 and Egr-3 mediate CD95L induction upon T-cell activation, for example, in the context of AICD. Moreover, Egr factors also contribute to CD95L expression in double-negative thymocytes. In mature T cells, Egr factors display different binding activities to Egr sites in the CD95L promoter of Th1 and Th2 cells, thereby potentially discriminating the two populations with regard to their AICD sensitivities. Upon cellular stress, the AP-1 complex, and upon growth factor withdrawal, the forkhead transcription factor, upregulate CD95L message and protein expression. 


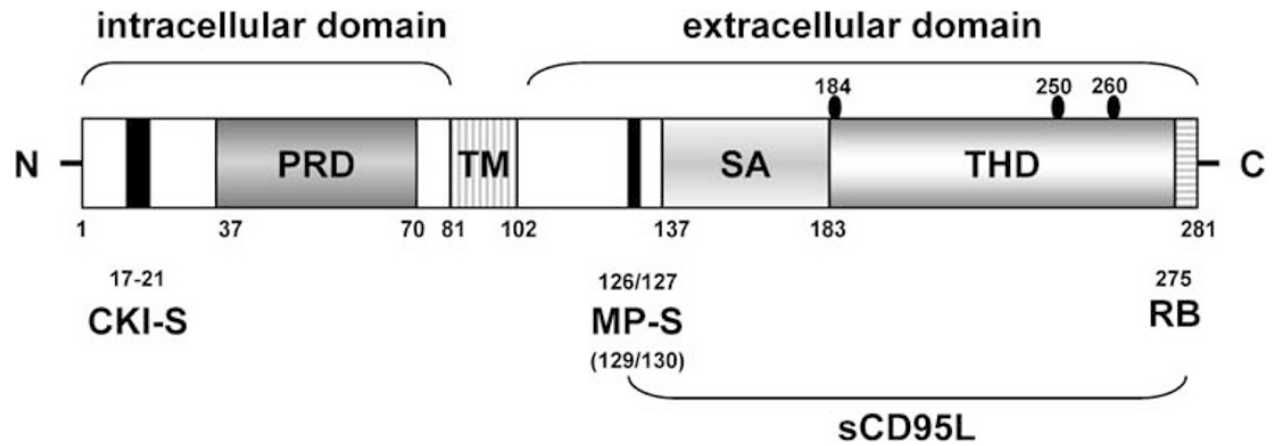

Figure 1 Structural features of a multifunctional death factor. Human CD95L is a type-II transmembrane molecule with 281 amino acids. The N-terminal cytoplasmic part (aa 1-81) contains a specific 'double' CKI substrate motif (CKI-S, aa 17-21). It also harbors an absolutely unique PRD (aa 37-70) which functions as a docking site for SH3 and WW domain proteins. The transmembrane region spans aa 81-102. Within the extracellular part, several metalloprotease substrate sites (MP-S) have been named to serve as cleavage sites for one or more metalloproteases (aa 109-115 (ELAELR), S126/127L and K129/130Q). These cleavage sites are located outside the region that is required for trimerization and self-assembly (SA). Thus, an SCD95L may also form trimers. The C-terminal THD (aa 183-281) contains several putative Nglycosylation sites (aa 184, 250, 260) and the very C-terminal receptor-binding site (RB) with a critical phenylalanin-residue at position 275.

\section{Soluble or Membrane CD95L}

The 'default' receptor for CD95L is CD95 (Fas, APO-1). Human CD95L-expressing cells require direct cell-to-cell contact to generate a death signal via the CD95 receptor (Figure 2). The CD95L/CD95-initiated death pathway has been studied and elucidated in great detail over the past decade (for review, see Nagata, ${ }^{14}$ Krammer ${ }^{15}$ and Peter and Krammer ${ }^{16}$ ). In addition, CD95L binding to an alternative decoy receptor 3 (DcR3, TNFRSF6B) was reported to inhibit CD95L function in several malignancies. Thus, the DcR3 gene was amplified in about half of 35 primary lung and colon tumours studied, and DcR3 message was detected in malignant tissue. ${ }^{17}$ Further, DcR3 is expressed by the majority of long-term and ex vivo malignant glioma cell lines, as well as in human glioblastoma in vivo. Chemotaxis assays revealed that in this context, DcR3 counteracts the chemotactic activity of CD95L against microglial cells in vitro. ${ }^{18}$ These findings suggest that by blocking CD95L function, DcR3 may be involved in the progression and immune evasion of malignant gliomas and other tumors.

Like other members of the TNF family, CD95L also comes in two flavors, a membrane-bound CD95L form (mCD95L) with an approximal molecular weight of $40 \mathrm{kDa}$ and a soluble CD95L variant (sCD95L) of 26-29 kDa. For both membrane and soluble CD95L, a spontaneous trimer formation has been suggested, which seems indispensable for triggering the death signal via CD95 oligomers. ${ }^{19}$ In CD95L-transfected COS cells, mono-, di- and trimeric forms were detected under nonreducing conditions. The ability of CD95L to self-associate was localized to a 47-amino-acid region in its extracellular domain (SA in Figure 1), and seems independent of transmembrane or cytoplasmic sequences. As previously shown for proteins such as the IL-6 receptor, theoretically, SCD95L can be generated by several independent mechanisms including differential splicing (ds), post-translational modification $(\mathrm{pm})$ or proteolytical cleavage $(\mathrm{pc})$. Indeed, Ayroldi et al. ${ }^{20}$ have been the first to describe a differentially spliced short variant of ds-sCD95L in unactivated spleen cells and hybridoma $T$ cells, which protected cells from CD95Linduced apoptosis. This splice variant lacks both the intracellular and the transmembrane parts of CD95L, and consists of a fraction of the extracellular domains encoded by exons 1 and 4 of the CD95L gene. To our knowledge, a pmsCD95L has not been reported.

The existence of the proteolytically cleaved soluble variant (pc-sCD95L) is widely accepted. It was initially identified as a biologically active molecule in supernatants of CD95L COS transfectants and stimulated T cells. Metalloproteinase (MP) inhibitors decreased the soluble and increased the membrane-bound fraction of CD95L. ${ }^{21}$ The putative MP cleavage site has been mapped to Lys129-GIn130 in murine cells and Ser126-Leu127 in human cells ${ }^{22}$ (Figure 1). These findings suggest that murine and human cells utilize different enzymes to produce SCD95L, or that one MP uses different cleavage sites. ${ }^{23}$ Since the MP which specifically cleaves TNF- $\alpha$ had been identified as a member of the 'a-disintegrin-andmetalloproteinase' (ADAM) MP family, and since the TNFhomologous portion of membrane-bound CD95L is similarly processed and shed, a closely related disintegrin has been suggested. Meanwhile, several candidate MPs have been analyzed in different cellular systems. Whereas CD95L cleavage could be achieved in some cells (i.e. glandular epithelial cells) by matrix metalloproteinase 7 (MMP$7=$ matrilysin), the MP(s) responsible for CD95L cleavage in other cell types remain to be identified. ${ }^{23}$ In a specialized population of murine epithelial cells, MMP-7 colocalizes with CD95L and cleaves off a biologically active (death-inducing) sCD95L. ${ }^{24}$ It was also reported that MMP-7 may play a role in the promotion of tumor survival and resistance to cytotoxic drugs (i.e. doxorubicin) by cleaving CD95L and reducing its effectiveness in triggering Fas-mediated apoptosis. Moreover, MMP-7 has been described to be highly expressed in pancreatic cancer precursors, regulating acinar-to-ductal metaplasia. ${ }^{25}$ Obviously, MMP-7 does not only cleave CD95L but also the receptor CD95. The sCD95 then antagonizes the effects of cytotoxic CD95L to result in resistance to CD95L-induced apoptosis in tumor cells. ${ }^{26}$

Taken together, it is clear that SCD95 is generated in different cell types by different means. However, the exact function of SCD95L as a death inducer or death inhibitor or even as a chemoattractant is still a matter of debate, and obviously depends on a given cellular microenvironment. Thus, it has been shown that human SCD95L is functional in 


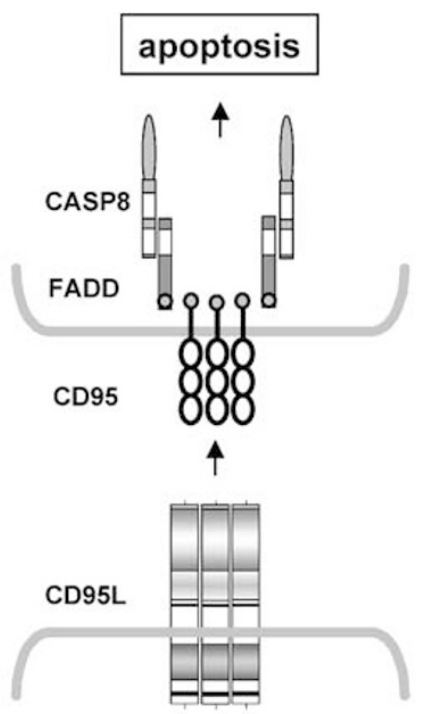

b

Signal 2' Signal $2 \quad$ Signal 1

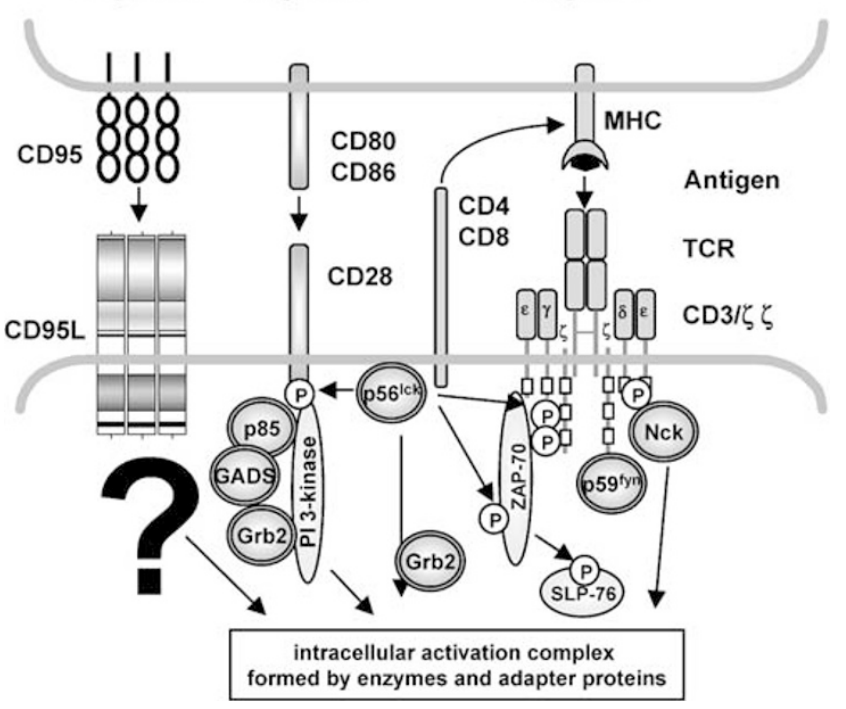

Figure 2 Dual function of CD95L. (a) CD95L trimers function as death factor to trigger CD95-induced apoptosis via FADD and caspase activation. This is the key event during activation-induced cell death. It plays a major role in target cell cytolysis, in the establishment of immune privilege and during tumor counterattack. (b) Model for the costimulatory function of CD95L. By virtue of assembling with a number of cytosolic $\mathrm{SH} 3$ domain proteins required for TCR-dependent activation (double-circled), CD95L could alter the TCR/CD3 stimulus in different ways. In such a scenario, CD95L may serve as a costimulatory molecule to either directly interfere with signal 1 given by the TCR or by interfering with signal 2 given by classical costimulatory molecules such as CD28. In addition, CD95L could also create signals independent of other surface receptors.

inducing apoptosis in presensitized cells at high doses even across species. In contrast, SCD95L has been shown to downregulate the apoptotic and inflammatory activity of its transmembrane counterpart in a number of cellular systems, suggesting that $\mathrm{mCD} 95 \mathrm{~L}$ is the functional molecule, and that shedding may counteract the cytotoxic activity of the membrane form. In terms of pathophysiology, SCD95L was found in the sera of patients with large granular lymphocytic leukemia (LGL) and NK cell lymphoma. ${ }^{27}$ Later reports pointed to a correlation of SCD95L serum levels with various other diseases, for example, the asymptomatic stage of HIV infection, rheumatoid arthritis and osteoarthritis, ulcerative colitis, Sjögren's Syndrome, acute respiratory distress syndrome (ARDS) and a large number of malignancies (Table 1). Consequently, SCD95L found in the serum of HIV-positive patients has been discussed to reflect the antiviral immune defense. ${ }^{28}$ Furthermore, CD95L shedding from the membrane might serve as a mechanism to adjust the killing activity of a T or NK cell. Interestingly, as soon as SCD95L interacts with fibronectin, cytotoxicity is restored, ${ }^{29}$ indicating rapid internalization of SCD95L after binding to its receptor. Therefore, it appears likely that the connection of SCD95L to cellmembrane or intercellular matrix proteins prevents internalization and enhances the probability to generate an effective death signal. Finally, it should be mentioned that for some cell types, for example, human neutrophils, SCD95L functions as a chemotactic factor. ${ }^{30,31}$

\section{The counterattack model}

As mentioned, various tumors have been suggested to constitutively express relatively high levels of mCD95L or secrete SCD95L. This could be regarded as a mechanism to ensure tumor survival by blocking immune defense. ${ }^{10,32-34}$ For example, Hahne et al. ${ }^{35}$ reported that melanoma cells escape immune elimination, although tumor-specific cytolytic $T$ cells directed against well-defined antigens are present in the population of tumor-infiltrating lymphocytes (TIL), or can even be detected in the peripheral blood of melanoma patients. When melanoma cells were analyzed for the expression of CD95L, both $\mathrm{mCD} 95 \mathrm{~L}$ and SCD95L could be detected, whereas normal melanocytes did not express CD95L. Since melanoma TIL mostly showed an activated phenotype expressing CD95, it was proposed that transformed melanocytes induce the apoptosis of CD95-sensitive TIL. As a proof of principle, CD95L-positive mouse melanoma cells led to rapid tumor formation when injected into normal mice, but to delayed tumor formation in CD95-deficient animals. In this regard, tumors might locally generate an immune-privileged microenvironment by attacking infiltrating T lymphocytes. Therfore, it was speculated that breaking this privilege or rendering TIL insensitive to CD95L-induced killing may provide a complementary approach in the therapy of malignant melanoma.

\section{CD95L and Disease - Other Pathophysiological Aspects}

The proof for a critical role of the CD95L molecule (and its receptor CD95) in the pathophysiology of autoimmune diseases was the analysis of generalized lymphoproliferative disease ( $g / d)$ mice, which was shown to carry a spontaneous loss-of-function point mutation in the CD95L locus. ${ }^{36}$ This mutation leads to the production of a nonfunctional protein and development of lymphadenopathy and splenomegaly with high titers of autoantibodies and defects in thymocyte maturation with a massive accumulation of CD4- and CD8- 
double-negative T cells. However, no corresponding CD95L mutation has been found to be associated with autoimmune disease in humans. Since only one of 75 patients with systemic lupus erythematosus (now classified as autoimmune lymphoproliferative syndrome (ALPS) 1B) was found to carry an 84-bp deletion within exon 4 of the CD95L gene resulting in a 28-amino acid in-frame deletion, it was considered that CD95L mutations are an uncommon cause of the disease. ${ }^{37}$ Interestingly, the study of peripheral blood mononuclear cells from this patient revealed decreased CD95L activity, decreased activation-induced cell death, and increased T-cell proliferation after activation. An involvement of a disturbed balance of CD95/CD95L interactions has also been postulated during organ-specific autoimmune diseases, such as Hashimoto's thyroiditis, insulin-dependent diabetes mellitus and multiple sclerosis. Furthermore, elevated levels of CD95L are thought to lead to the loss of virus-specific CTL observed during HIV infection, or to reduction of the antitumor activity of TIL in many tumors (see also Table 1).

Outside the immune system, CD95L has also been implicated, for example, in the pathology of toxic epidermal necrolysis (TEN) by mediating keratinocyte apoptosis. ${ }^{38}$ In the sera of patients suffering from TEN, high levels of soluble CD95L were detected, and CD95L produced by keratinocytes of TEN patients induced keratinocyte cell death. Incubating keratinocytes with intravenous immunoglobulin (IVIG) containing a naturally occurring anti-CD95 immunoglobulin completely inhibited CD95-mediated keratinocyte apoptosis. In TEN patients treated with IVIG, progression of skin disease was rapidly reversed. ${ }^{38}$

The CD95L/CD95 system also contributes to the control of infection. Grassme et al. ${ }^{39}$ showed that Pseudomonas aeruginosa infection induces the apoptosis of lung epithelial cells by activation of the endogenous CD95/CD95L system only in CD95/CD95L wild type but not in deficient mice. Thus, inflammation and sepsis after $P$. aeruginosa infection was seen in mutant but not in normal mice - another example that controlled cell death and elimination by apoptosis is required to prevent inflammatory complications. One example for an obvious strategy of viruses to evade an immune response was reported by Raftery et al. ${ }^{40}$ They observed that besides the downregulation of a number of accessory and costimulatory molecules (MHC I, CD40, CD86) on infected dendritic cells, some CMV strains further compromise the immune system by inducing the expression of death molecules such as TRAIL and CD95L. This upregulation of apoptosis-inducing ligands will not only suppress the T-cell response, but also delete activated T cells. However, although elevated $(>50 \mathrm{pg} / \mathrm{ml}$ ) levels of sCD95L have been observed also under various other pathological conditions in human sera, the clinical significance is still unclear. ${ }^{14}$

\section{CD95L in T Lymphocytes}

During T-cell development and maturation, CD95L was supposed to be involved in negative selection. In this context, CD95L is propagated by some investigators as an effector molecule leading to the deletion of autoreactive thymocytes. $^{41-43}$ However, the role of CD95L is discussed controversially also in this context, and from studies in transgenic mice the clear statement was that the CD95/ CD95L system is involved in peripheral but not thymic deletion of T lymphocytes. ${ }^{2,44}$ In mature T cells, CD95L is differentially expressed in individual T-cell subsets. On CD4 ${ }^{+}$cells, CD95L appears as a membrane-bound receptor on T helper 1 (Th1) and only on some T helper 2 (Th2) cells following T-cell receptor (TCR) stimulation by antigen, anti-TCR/CD3 antibodies, mitogenic lectins, bacterial superantigens or other stimuli such as phorbol esters and calcium ionophore treatment. In such cells, CD95L is a major effector molecule in the course of AICD, thereby regulating the termination of $\mathrm{T}$ cell responses, peripheral deletion and preventing autoimmunity. For Th2 cells, a reduced expression or absence of CD95L under similar conditions has been reported, which explains the resistance of such clones to AICD. ${ }^{45}$ However, further mechanisms have been suggested to render these cells unresponsive to CD95-mediated cell death. Some reports indicate a role for FAP1, the Fas-associated phosphatase 1 and others a role of FLIP $_{\text {short }}$ or $\mathrm{Bcl}_{\mathrm{xl}}$ in the generation of Th2 resistance. ${ }^{46}$ In cytotoxic T cells (and NK cells), CD95L is synthetized upon primary stimulation and then directed to and stored in secretory granules/lysosomes. ${ }^{47}$ Besides CD95L, this type of lysosomes also contain perforins and granzymes. Upon contact, for example, with a virally infected target cell, the TCR-derived signal will lead to a redistribution of cytoskeletal elements, resulting in extrusion of the cytotoxic molecules into the immunological synapse ${ }^{48}$ accompanied by surface expression of $\mathrm{mCD} 95 \mathrm{~L}$ and binding to CD95 on the target cell or shedding of SCD95L.

\section{CD95L as a Costimulator for T Cells}

A series of reports employing mice defective for CD95L ( $g / d)$ or CD95 (lpr, Ipr $\left.{ }^{c g}\right)$ suggest a costimulatory function for CD95L in both $\mathrm{CD}^{+}$and $\mathrm{CD}^{+}{ }^{+} \mathrm{T}_{\text {cells. }}{ }^{49-52}$ While $\mathrm{CD}^{+}{ }^{+}$cells showed a reduction in TCR/CD3-induced cell cycle progression and entered apoptosis, $\mathrm{CD}^{+}$cells required the CD95L costimulation to rapidly progress through the cell cycle, resulting in proliferation and acquisition of cytolytic effector function. Although the molecular basis for the reverse costimulatory signalling of CD95L remains unknown, two motifs present in the cytosolic region of CD95L are likely to be involved in the regulation of receptor crosstalk and the reverse signalling: a casein kinase I (CKI) substrate motif and a proline-rich domain (PRD) (Figure 1).

\section{CD95L and Other Bidirectional Signal Transducers with CKI Substrate Motifs}

Most ligands of the tumor necrosis factor (TNF) superfamily are transmembrane proteins that share structurally related extracellular TNF homology domains (THD), which bind to cystein-rich domains (CRD) of their specific receptors (of the TNF-receptor superfamily). In general, ligand/receptor interactions of members of these families regulate key events in cellular activation, proliferation, differentiation, cell death and survival of immune cells and other tissues. ${ }^{53}$ Since most of the TNF family members act as membrane-bound factors and 
require direct cell-to-cell contact, it seems obvious that signalling upon ligation triggers cellular responses in both directions. Substantial in vivo evidence for the physiological role of reverse signalling has been shown for CD40L, but bidirectional (i.e. costimulatory) functions have also been ascribed to several other members of the TNF superfamily in $T$ cells, B cells and monocytes (for other individual references, see Locksley et al. ${ }^{53}$ and Wenzel et al. ${ }^{54}$ ). Thus, the ligand/ receptor complexes of OX40L/CD134 (TNFSF4/TNFRSF4), CD154/CD40 (TNFSF5/TNFRSF5), CD70/CD27 (TNFSF7/ TNFRSF7), CD153/CD30 (TNFSF8/TNFRSF8), 4-1BB-L/ CD137 (TNFSF9/TNFRSF9), TRANCE/RANK (TNFSF11/ TNFRSF11), LIGHT/LIGHTR (TNFSF14/TNFRSF14) ${ }^{55-57}$ and the death factors TNF, TRAIL (TNFSF10) ${ }^{58}$ and CD95L (TNFSF6) were reported to convey retrograde signals. Of interest, so far the bidirectional signalling capacity has been mostly associated with those six of 16 TNF family members (CD27L, CD30L, CD40L, CD137L, TNF $\alpha$ and CD95L) that contain a putative casein kinase I (CKI) substrate motif (SXXS-). Since the corresponding phosphorylation sites are not present in any of the remaining TNF family members, it seems likely that reverse signalling depends on this domain. ${ }^{59}$ However, so far, CKI-dependent serine phosphorylation upon receptor activation has only been shown for TNF- $\alpha$ but none of the other ligands. In the case of CD95L, the membrane distal $\mathrm{N}$-terminus in fact contains a unique 'double' CKI substrate site (amino acids 17-21 in man (-SSASS-) and 17-22 in mice (-SSATSS-)). The presence of such a motif (-S(P)XXS-) indicates a role for an additional kinase to regulate the sensitivity of this site itself for CKI by phosphorylation. ${ }^{59}$ Nevertheless, no experimental data are available to prove the involvement of any kinase or phosphatase in the regulation of CD95L reverse signal transduction.

\section{PRD as a Protein-Docking Site of CD95L}

The second prominent signalling motif within the cytosolic region of $C D 95 L$ is a PRD spanning amino acids 37-70 of the human CD95L (Figure 1). This PRD is unique among the members of the TNF family, and certainly plays a major role in the biology of CD95L. When CD95L was cloned and sequenced, the PRD was thought to function as a docking site for proteins that regulate signalling, trafficking and expression of CD95L. After 1 year, it was found that peptides corresponding to the proline-rich region of the murine CD95L (aa 40-77) were able to selectively interact with the $\mathrm{SH} 3$ domains of the Src-related kinase $\mathrm{p} 59^{\mathrm{fyn}(\mathrm{T})}{ }^{60}$ From this it was suggested that CD95L membrane expression is primarily regulated through an activation-independent SH3-mediated association with p59 $9^{\mathrm{fyn}(\mathrm{T})}$. In 1996, Hachiya and co-workers deposited several protein fragments in the NCBI database, which were found to interact with CD95L in a yeast two-hybrid screen. Although these 'Fas-ligand associated factors' (FLAF1-3-AAB93495, AAB93496, AAB93497) contained either a WW domain (characterized by two tryptophane (W) residues spaced in a certain distance) or one or more $\mathrm{SH} 3$ (Src homology 3) domains as the PRD-interacting region, and were supposed to regulate CD95L stability, no further results were published. Sequence comparison reveals that FLAF-1 forms part of the formin-binding protein 11 (FBP11, also called the huntingtin-interacting protein HYPA), FLAF2 is part of the c-Cbl-associated protein SH3P12='sorbin and SH3 domain containing 1' (containing 3 SH3 domains), and FLAF3 represents a portion of the BAl1-associated protein 2 (BAP2)-beta (with a single SH3 domain).

\section{Association of Proteins with Proline Recognition Domains}

In order to identify proteins that interact with the PRD of CD95L cytoplasmic tail, we used two different protein-based approaches. First, we screened a large panel of $\mathrm{SH} 3$ and WW domains expressed as GST fusion proteins for their ability to precipitate CD95L from stable transfectants and $T$ lymphoblasts. ${ }^{54}$ Second, we constructed fusion proteins that contain the cytosolic part of CD95L with or without proline stretch, and used these for pull-down assays from T-cell lysates. ${ }^{61}$ Our analyses revealed that in addition to Fyn SH3 domains, a number of other $\mathrm{SH} 3$ or WW modules are also capable of effectively and specifically interacting with the full-length CD95L overexpressed in transfectants and inducibly expressed in stimulated human $\mathrm{T}$ lymphocytes. The identification of various other Src-related kinases and different adapter proteins of the growth factor receptor-binding protein 2 (Grb2) family, phosphoinositol 3 kinase (PI 3-kinase), Nck and Phox47 and the WW domain proteins FE65, FBP11 and dystrophin as putative CD95L-interacting molecules, opens new insights into the potential of $\mathrm{CD} 95 \mathrm{~L}$ to regulate and modulate activation processes in T cells and other CD95L-expressing cells. It should be stressed, however, that not all SH3 or WW domains bind to CD95L, and if there are more than one $\mathrm{SH} 3$ domains in a given protein, binding is not seen for all domains. Moreover, many $\mathrm{SH} 3$ domains that we tested in our screening assay did not show any binding to CD95L, and not all SH3 domain-containing proteins that we found to interact with CD95L in vitro are present, for example, in T cells indicating a cell-type-specific set of interactors and interactions regulating CD95L biology in a given cell type.

\section{Speculations about Reverse Signalling in T Cells}

For T cells, the role of Src-related kinases (i.e. Lck, Fyn and Yes), PI 3-kinase, adapter proteins including Grb2, Gads and Nck-1 in activation has been extensively investigated over the past two decades. Most of these molecules mediate critical steps in the assembly of activation complexes associated with antigen receptors, adhesion molecules and costimulatory downstream signalling mediators. Since we found Grb2 in precipitates with CD95L fusion proteins, this interaction may serve as a model scenario for CD95L-to-TCR crosstalk (Figure 2b). Grb2 is a well-defined adapter protein consisting of two $\mathrm{SH} 3$ domains flanking a single $\mathrm{SH} 2$ domain that plays a pivotal role in signalling of the insulin receptor, growth factor receptors, the TCR and others. TCR ligation rapidly results in recruitment of the Grb2-Sos complex to the phosphorylated transmembrane adapter LAT (linker for activated T cells), thereby triggering the Ras/MAPK pathway, leading to 
transcription of a number of proteins required for T-cell activation and differentiation. ${ }^{62}$ An interaction of Grb2 and CD95L could therefore point to a competitive or synergistic crosstalk between CD95L and the TCR, and explain the increased proliferation described for $\mathrm{CD}^{+}$cells in vivo. ${ }^{50,52}$ Since the Grb2-Sos complex is well known to recruit the small G-protein Ras, and since spatial proximity between Ras and the active p110 subunit is crucial for PI-3K activation, Grb2 might not only directly provide a stimulus for proliferation (as has been shown for the EGF-R-pathway), but also faciliate PI$3 \mathrm{~K}$ activation. The $\mathrm{PI}-3 \mathrm{~K}$ product $\mathrm{PIP}_{3}$ was shown to activate both the $\mathrm{PIP}_{3}$-dependent kinase 1 (PDK-1) and protein kinase $B(P K B) / A K T$, resulting in antiapoptotic signals in skin tumor cells via phosphorylation of Bad or the transcription factor FOXO3a. Taken together, Grb2 and the p85 adapter subunit of PI-3K, both binding to the PRD via their SH3 domains, might generate both a proliferative (via Ras and MAP-kinase) and antiapoptotic (via PKB) signal at the same time, regardless of whether the CD95L extracellular domain is cleaved from the surface or not.

Our own and other laboratories' experiments to prove a direct involvement of CD95L in the regulation of TCR/CD3 responses as a costimulatory molecule turned out to become puzzling. We failed to detect changes in tyrosine phosphorylation, Erk activation and AKT activation as a direct action of CD95L ligation. We also failed to detect CD95L-dependent changes in TCR/CD3 or phorbolester/ionophore stimulation of activated T cells. However, the antibodies and the CD95Fc fusion protein used in our studies are functional, for example, in blocking AICD in the very same clones and lines. In addition, we reproducibly observe an inhibitory effect of CD95L crosslinking on the proliferative capacity of freshly isolated peripheral blood $\mathrm{T}$ cells upon stimulation with anti$\mathrm{CD} 3 \mathrm{mAb}$ or superantigen. Nonetheless, the exact experimental conditions to biochemically identify the pathways of CD95L reverse signalling still need to be worked out. Such analyses are further hampered by the fact that the regulation of CD95L expression is far more complex and dynamic than expected, and that $\mathrm{CD}^{+}$and $\mathrm{CD}^{+}{ }^{+}$or Th1 and Th2 cells differ in their expression patterns. Therefore, upcoming experiments will focus on the use of stably transfected CD95L T-cell lines established in our laboratory, on the mechanisms of inhibition of primary T-cell stimulation and on the role of oligomerization induced by crosslinking of CD95L.

\section{Nck-linking CD95L, the Cytoskeleton and the TCR/CD3 Complex}

We recently also described the interaction of two of three $\mathrm{SH} 3$ domains of Nck-1 with the PRD of CD95L. ${ }^{54}$ These data were now confirmed by cotransfection, co-precipitation and colocalization studies with wild type and mutants of Nck-1 in different cell lines (Qian et al., manuscript in preparation). Nck-1 is a classical adapter protein of 377 amino acids, which is built of one c-terminal $\mathrm{SH} 2$ and three $\mathrm{SH} 3$ domains. It was shown to constitutively associate with the active $\gamma 2$ isoform of the serine/threonine casein kinase I $(\mathrm{CKI}-\gamma 2),{ }^{63}$ and could therefore be involved in sequestering the kinase to the substrate CD95L. As mentioned, Watts et al. ${ }^{59}$ described the CKI-dependent phosphorylation of a corresponding motif in the TNF-R. Similarly, serine phosphorylation by a casein kinase recruited via Nck-1 could be responsible for reverse signalling through CD95L. Nck-1 is also implicated in regulation of the cytoskeleton through interaction with the Wiskott-Aldrich syndrom-protein (WASP), the WASP interacting protein (WIP), WASP-family verprolin homologous protein (WAVE1), the Centaurin- $\alpha$ family of PIP3-binding proteins, the p21-activated kinase (PAK1), the $\varepsilon$-chain of CD3 and others. The cytoskeletal association of Nck-1 may also play a role in CD95L transport to the cell membrane (Figure 3, see below).

\section{PACSIN and FBP17 as Interactors for CD95L Models and Hypotheses}

We used variants of the intracellular region of human CD95L with or without PRD, expressed as GST fusion proteins to precipitate binding partners from T-cell lysates. The precipitates were separated by two-dimensional gel electrophoresis and sequence information obtained by MALDI-TOF-based peptide finger-print analyses. As already mentioned, we verified the Grb2 association reported earlier. Besides, with PACSIN2 and FBP17, we identified two structurally related proteins that are likely to be involved in protein trafficking and cytoskeletal organization, and may also be responsible for the presence of $\beta$-tubulin and actin in the same precipitates. ${ }^{61}$

PACSIN2 is a $53 \mathrm{kDa}$ human splice variant of the rat Syndapin II, and a member of a family of cytosolic adapter proteins that link clathrin-dependent endocytosis to the actin cytoskeleton, and are involved in the regulation of vesicular traffic. $^{61}$ To date, three PACSINs are known, all of which contain phosphorylation sites for casein kinase II and protein kinase $C$ (the name PACSIN refers to 'protein kinase $C$ and casein kinase substrate in neurons'). In contrast to the ubiquitously expressed PACSIN2, PACSIN1 was not detected in cells other than neurons. In terms of function, it was recently reported that PACSIN isoforms specifically interact with Sos, thereby regulating actin dynamics via MAP kinase signalling. In the context of CD95L, PACSIN2 could be involved in the reorganization of the cytoskeleton and in trafficking of cytoplasmic vesicles, leading to storage and regulation of extrusion of CD95L or to endocytosis, recycling or degradation. The potential association of PACSINs with the GEF Sos could also suggest a role of this interaction in downstream signalling to MAP kinases. Most interestingly, the activity of the sheddase ADAM13 that contains three prolinerich repeats in its long cytoplasmic tail was shown to be efficiently inhibited by the SH3 domain of PACSIN2 in vivo. However, due to the presence of only one $\mathrm{SH} 3$ domain per PACSIN2 molecule, CD95L and ADAM13 should either compete for this docking site, or are connected via multimerization of PACSIN2, probably involving the coiled-coil domain.

Formin-binding protein 17 (FBP17), a $72 \mathrm{kDa}$ protein, belongs to the heterogeneous family of formin-binding proteins, which, in association with the various formins, also regulate the organization and assembly of the actin cytoskeleton, and are involved in orchestrating cell motility, adhesion 


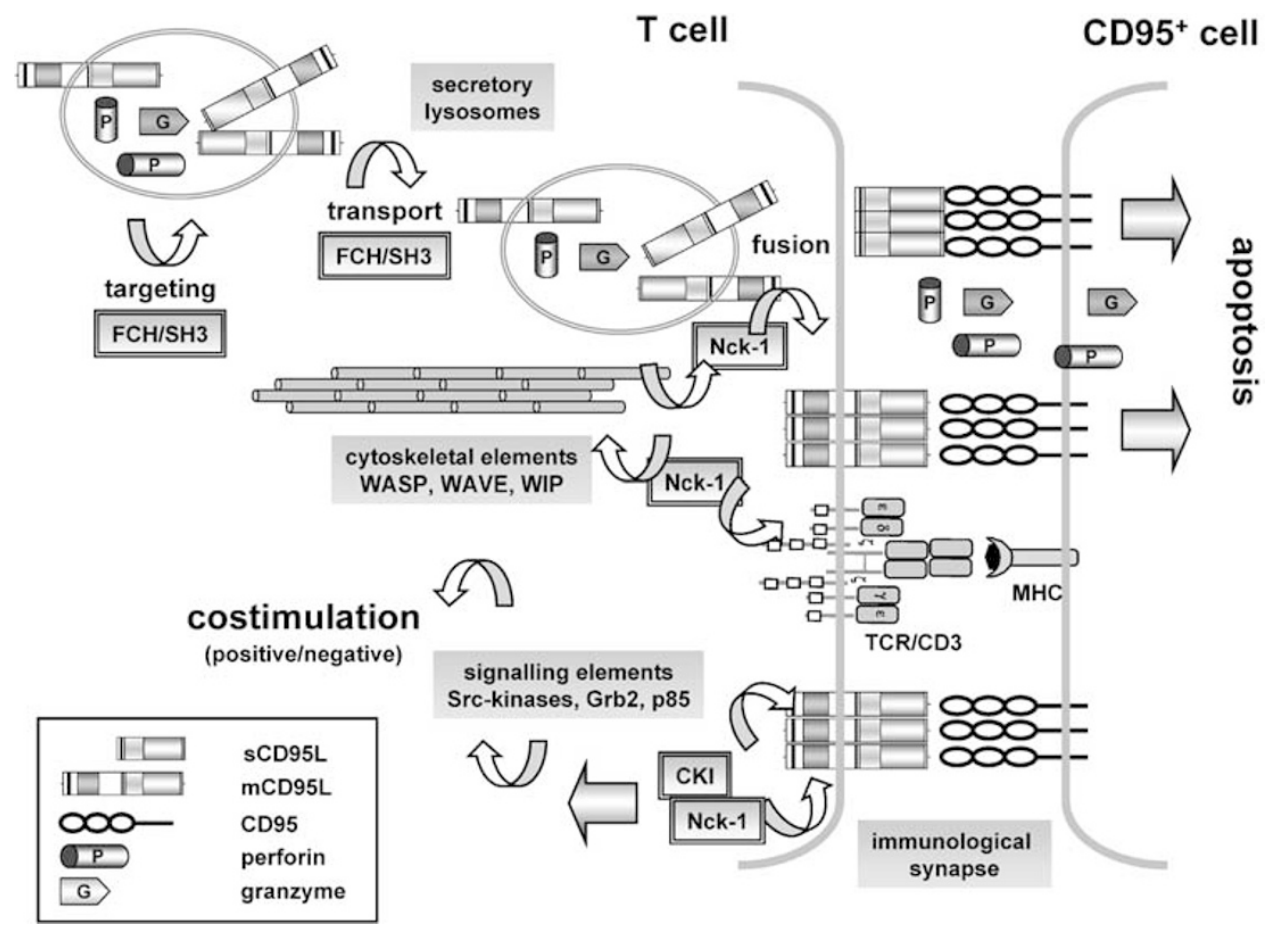

Figure $3 \mathrm{CD} 95 \mathrm{~L}$ interacting proteins - facts and hypotheses. Recent work by Griffiths et al. ${ }^{47,48,71}$ showed that in cytotoxic cells, CD95L is targeted to secretory granules/lysosomes. Besides CD95L, these lysosomes contain other cytotoxic molecules such as granzymes and perforins, which in concert contribute to an effective target cell lysis. Targeting of CD95L to these lysosomes strictly depends on the PRD within the cytoplasmic tail of the molecule. In this context, members of the FCH/SH3 family (including FBP17, PACSINs, CD2BP1 and others) are good candidates for directing the localization and transport of CD95L (and/or secretory lysosomes in general). All proteins of this family are involved in actin cytoskeleton reorganization and protein/organelle trafficking. Other interactors for CD95L are the small adapter proteins Grb2 and Nck-1. Therefore, a working model for CD95L function would be that the PRD is not only required for lysosomal targeting, but also for transport and fusion of these lysosomes to the area where the immunological synapse is formed. This could be followed by the release of the various cytolytic factors and in the presence of a MMP (such as matrilysin, MMP-7) by CD95L cleavage. Whether the SCD95L induces or inhibits apoptosis then depends on the potential to form active multimers. The fact that CD95L has been found to act as a 'negative' or 'positive' costimulatory molecule for T cells underscores the complex biology of the molecule. Whether the same (FCH/SH3-family, Grb2 or Nck-1) or other (e.g. CKI, PI 3-kinases or Src kinases) CD95L interacting molecules are involved in this aspect, remains open.

and cytokinesis. ${ }^{64}$ Interestingly, PACSINs and FBP17 are very similar with regard to their overall modular composition. Both contain a C-terminal $\mathrm{SH} 3$ domain (that interacts with CD95L) and an N-terminal FCH (Fes/CIP homology) domain that shares homology, for example, to regulators of the actin cytoskeleton in yeast. Fuchs et al. ${ }^{65}$ showed that FBP17 is widely expressed in a variety of human tissues. They demonstrated the interaction of FBP17 with sorting nexin (SNX2), and thus provided a link between MLL, a gene associated with myelogenous leukemia, and the epidermal growth factor receptor (EGFR) pathway. In general, SNXproteins are molecules crucially involved in directed protein trafficking and lysosomal targeting. ${ }^{66}$ Therefore, association of CD95L with FBP17 could represent an alternative mode of lysosomal transport of CD95L, and also point to a signalling crosstalk between the death factor and the EGFR pathway. As mentioned, we also found actin and $\beta$-tubulin in pulldown precipitates. Although we did not yet formally prove the interactions, this could provide more evidence for the association of CD95L with lysosomes and/or cytoskeletal elements, since $\beta$-tubulin is a major component of microtubules, arranging the mitotic spindle during cell division and regulating axonal transport and organelle positioning. In addition, it was reported that tubulin plays an important role in G-protein-mediated signal transduction. Obviously, even though tubulins are structural proteins, they participate in cellular signalling albeit through physical forces. In this scenario, it is discussed whether tubulins modulate the conformation of receptors or $\mathrm{G}$-proteins, either leading to a change in receptor-ligand interaction and/or influencing the G-protein-mediated signal.

PACSIN2 and FBP17 belong to a larger family of structurally related proteins, which, as mentioned, are in most cases associated with lysosomal transport processes, cytoskeletal organization and signal transduction. At least nine members of this family are known in humans. They all share an overall modular composition with an N-terminal FCH (Fes/ CIP4 homology) domain, a central coiled-coil region and a Cterminal SH3 domain. Interestingly, SH3 domains of all other members of this protein family (PACSIN1 and PACSIN3, CD2-binding protein 1 (CD2BP1=PSTPIP), RhoGAP C1 and Rho GAP C1-related protein, FLJ00007 protein and CDC42interacting protein CIP4), bind to CD95L in vitro (Qian et al., unpublished; Baum and Zörnig, unpublished). 


\section{Targeting of CD95L to secretory lysosomes}

The phenomena associated with CD95L, namely surface expression on $\mathrm{CD}^{+}{ }^{+} \mathrm{T}$ cells and lysosomal expression and transport associated with cytotoxic $\left(\mathrm{CD} 4^{+}\right.$or $\left.\mathrm{CD}^{+}\right) \mathrm{T}$ cells and NK cells, may be regulated differentially, and thus require distinct sets of CD95L-binding proteins. In both cases, the association with regulatory elements seems to depend on the PRD of the cytosolic portion of the molecule. ${ }^{67}$ It has been suggested that sorting of CD95L from the Golgi network to secretory lysosomes may provide a general mechanism for controlling the cell surface appearance of proteins involved in immune regulation. The association of membrane-bound or

Table 1 Examples for disease-association of sCD95L expression

\begin{tabular}{ll}
\hline Disease & sCD95L in patient's sera \\
\hline (a) Cancers & $\begin{array}{l}\text { Significantly increased } \\
\text { (mCD95L also increased) }\end{array}$ \\
Colon adenocarcinoma & $\begin{array}{l}\text { Significantly increased } \\
\text { (mCD95L also increased) }\end{array}$ \\
NK cell lymphoma & $\begin{array}{l}\text { Significantly increased } \\
\text { (mCD95L also increased) } \\
\text { Unchanged }\end{array}$ \\
Melanoma & Unchanged \\
Multiple myeloma & Significantly increased \\
Bladder cancer &
\end{tabular}

\section{Reference}

(b) Immunologic diseases Graves' hyperthyroidism

Multiple sclerosis

Graft-versus-host-disease

Sjögren's syndrome

Churg-Strauss vasculitis

(c) Infectious diseases

Viral liver cirrhosis

Pulmonary sarcoidosis

Human immunodeficiency virus infection

Helicobacter-pylori-positive gastric ulcer

Acute pancreatitis

(d) Other diseases

Atherosclerosis in

hypertensive patients

Acute myocardial infarction

Acute respiratory distress

Syndrome (ARDS)

Fibrosing lung disease

Severe traumatic brain injury

Rheumatoid arthritis/

osteoarthritis

Histiocytic necrotizing

lymphadenitis

Alzheimer's disease

Severe acute alcoholic hepatitis and alcoholic cirrhosis

Hepatic failure
Significantly increased

Significantly decreased

Significantly increased

Significantly increased

Significantly increased (mCD95L decreased)

Significantly increased

Significantly increased

Significantly increased in asymptomatic stage Significantly increased

Significantly decreased

Significantly increased

Significantly increased

Significantly increased

Significantly increased

Prolonged intrathecal release

Increased in synovial fluid

Significantly increased

Not investigated

Significantly increased

Significantly decreased
Song $\mathrm{E}$ et al. (2001) $\mathrm{Br} \mathrm{J}$

Cancer 85: 1047

Tanaka M et al. (1995)

EMBO J. 14: 1129

Tanaka M et al. (1995)

EMBO J. 14: 1129

Melzani G et al. (2002)

Dermatology 205: 111

Kanda $Y$ et al. (2001) Leuk.

Lymphoma 40: 351

Mizutani $Y$ et al. (2001)

Cancer 92: 287

Wang CY et al. (2002)

Metabolism 51: 769

Macchi B et al. (2001)

Neuroreport 12: 4113

Kanda Y et al. (1998) Bone

Marrow Transplant. 22: 751

Ishimaru $\mathrm{N}$ et al. (2001) J.

Immunol. 167: 6031

Muschen M et al. (1999) Am.

J. Pathol. 155: 915

Wang PS et al. (2002) Lung

180: 25

Shikuwa C et al. (2002)

Respiration 69: 242

Bahr GM et al. (1997) Blood

90: 896

Shimada M et al. (2002)

Scand. J. Gastroenterol. 37:

501

Endo S et al. (2000) Res.

Commun. Mol. Pathol.

Pharmacol. 108: 179

Okura T et al. (2002) J.

Hypertens. 20: 895

Shimizu M et al. (2002) J.

Am. Coll. Cardiol. 39: 585

Matute-Bello G et al. (1999)

J. Immunol. 163: 2217

Kuwano K et al. (2002)

Respirology 7: 15

Lenzlinger PM et al. (2002)

J. Neuroimmunol. 122: 167

Hashimoto $\mathrm{H}$ et al. (1998)

Arthritis Rheum. 41: 657

Kato K et al. (2001) Int. J.

Hematol. 73: 84

Tourneur L et al. (2001) J.

Immunol. 167: 1338

Taieb J et al. (1998) Lancet

351: 1930

Nakae H et al. (2001) J. Crit. Care 16: 59 
intracellular CD95L to proline-interacting adapter proteins or enzymes may therefore not only explain the crosstalk between the death factor and other surface receptors. Of note, also in this context, the CD95L-associated SH3 adapters of the Grb2 family and especially Nck-1 have crucial roles for the formation of the immunological synapse upon TCR ligation, and the link to cytoskeletal reorganization. ${ }^{68}$

\section{Secretory lysosomes and the 'kiss of death'}

The specialized lysosomal compartment where CD95L is sorted to contains granzymes $A, B, H, K$ and $M$, pore-forming perforin monomers and, as a transmembrane component, $\mathrm{CD} \mathrm{L}^{69}$ (Figure 3). Upon interaction with a target cell, the lipid bilayer of the secretory lysosomes fuses with the plasma membrane, thereby releasing the soluble lysosome components and presenting mCD95L on the cell surface. This trigger of target cell apoptosis has been referred to as the 'kiss of death'. ${ }^{70}$ Unlike other proteins that are sorted to the lysosomal compartment by di-leucine or tyrosine-based sorting motifs, Griffiths et al. ${ }^{67}$ convincingly showed that CD95L is targeted to secretory lysosomes via the PRD. This study proposed a new model for sorting pathways in cells with secretory lysosomes compared to conventional lysosomes. Upon transfection, the presence of the PRD was sufficient to sort CD95L to secretory lysosomes, whereas deletion of the PRD, but not of tyrosine or di-leucine motifs in the tail of CD95L (7Y-9Y-13Y and 29V$30 \mathrm{~L})$, resulted in surface expression of the protein. ${ }^{67}$ As detailed earlier, various tumors have been suggested to constitutively express high levels of CD95L on their cell membrane, as a mechanism to effectively kill TILs by a mechanism termed the 'Fas counterattack'. ${ }^{10,32,34}$ One example that also highlights the targeting of CD95L to specific subcellular lysosomal compartments is the identification of CD95L-loaded melanosomes. ${ }^{33}$ In these structures, CD95L colocalizes with both melanosomal and lysosomal antigens. Isolated melanosomes express CD95L and exert CD95mediated apoptosis in Jurkat cells. The mechanisms leading to expression and storage or secretion of CD95L in such tumor cells have not yet been investigated. It will be interesting to find out whether alterations or mutations occuring in those malignancies might be related to mutations in the PRD of CD95L, or any of the transport proteins involved. But, although a number of possible interaction partners of CD95L have been identified, the exact regulatory molecules guiding CD95L either to the secretory lysosomes or the plasma membrane remain unknown.

\section{Conclusions}

Although the CD95L has been identified 10 years back, we are still far from understanding the complex biology of the molecule. CD95L is found in both immunologically active and immune-privileged tissues and in immune cells as well as in tumor cells. The regulation of expression and the function seem to be cell-type-specific and microenvironment-dependent. This concerns the cytotoxic function and the potential costimulatory signalling capacity. To monitor a reverse signalling in vitro proved to be a frustrating experience. However, as has been demonstrated for TNF, the upcoming analyses of the CKI substrate motif may shed light on this function that has been clearly demonstrated in vivo. In addition, the role of the soluble form is unclear, and it is not known under which (pathological) conditions it is produced. In terms of CD95L transport and storage, the identification of the PRD interacting proteins will help to position the molecule in the complex cytoskeleton and transport network.

\section{Acknowledgements}

\#OJ is supported by grants from the Deutsche Forschungsgemeinschaft (SFB 415) and the Faculty of Medicine, University of Kiel.

\section{References}

1. Suda T, Takahashi T, Golstein P and Nagata S (1993) Molecular cloning and expression of the Fas ligand, a novel member of the tumor necrosis factor family. Cell 75: 1169-1178

2. Green DR, Droin N and Pinkoski M (2003) Activation-induced cell death in T cells. Immunol. Rev. 193: 70-81

3. Eischen CM, Schilling JD, Lynch DH, Krammer PH and Leibson PJ (1996) Fc receptor-induced expression of Fas ligand on activated NK cells facilitates cellmediated cytotoxicity and subsequent autocrine NK cell apoptosis. J. Immunol. 156: 2693-2699

4. Kiener PA, Davis PM, Rankin BM, Klebanoff SJ, Ledbetter JA, Starling GC and Liles WC (1997) Human monocytic cells contain high levels of intracellular Fas ligand: rapid release following cellular activation. J. Immunol. 159: 1594-1598

5. De Maria R, Zeuner A, Eramo A, Domenichelli C, Bonci D, Grignani F, Srinivasula SM, Alnemri ES, Testa U and Peschle C (1999) Negative regulation of erythropoiesis by caspase-mediated cleavage of GATA-1. Nature 401: 489493

6. Kaplan HJ, Leibole MA, Tezel T and Ferguson TA (1999) Fas ligand (CD95 ligand) controls angiogenesis beneath the retina. Nat. Med. 5: 292-297

7. Hill LL, Ouhtit A, Loughlin SM, Kripke ML, Ananthaswamy HN and OwenSchaub LB (1999) Fas ligand: a sensor for DNA damage critical in skin cancer etiology. Science 285: 898-900

8. Pinkoski MJ and Green DR (1999) Fas ligand, death gene. Cell Death Differ. 6: 1174-1181

9. Restifo NP (2000) Not so Fas: re-evaluating the mechanisms of immune privilege and tumor escape. Nat. Med. 6: 493-495

10. O'Connell J, Houston A, Bennett MW, O'Sullivan GC and Shanahan F (2001) Immune privilege or inflammation? Insights into the Fas ligand enigma. Nat. Med. 7: 271-274

11. Ferguson TA, Green DR and Griffith TS (2002) Cell death and immune privilege. Int. Rev. Immunol. 21: 153-172

12. Nakajima M, Nakajima A, Kayagaki N, Honda $M$, Yagita $H$ and Okumura $K$ (1997) Expression of Fas ligand and its receptor in cutaneous lupus: implication in tissue injury. Clin. Immunol. Immunopathol. 83: 223-229

13. Kavurma MM and Khachigian LM (2003) Signaling and transcriptional control of Fas ligand gene expression. Cell Death Differ. 10: $36-44$

14. Nagata S (1999) Fas ligand-induced apoptosis. Annu. Rev. Genet. 33: 29-55

15. Krammer PH (1999) CD95(APO-1/Fas)-mediated apoptosis: live and let die. Adv. Immunol. 71: 163-210

16. Peter ME and Krammer PH (2003) The CD95(APO-1/Fas) DISC and beyond. Cell Death Differ. 10: 26-35

17. Pitti RM, Marsters SA, Lawrence DA, Roy M, Kischkel FC, Dowd P, Huang A, Donahue CJ, Sherwood SW, Baldwin DT, Godowski PJ, Wood WI, Gurney AL, Hillan KJ, Cohen RL, Goddard AD, Botstein D and Ashkenazi A (1998) Genomic amplification of a decoy receptor for Fas ligand in lung and colon cancer. Nature 396: 699-703

18. Roth W, Isenmann S, Nakamura M, Platten M, Wick W, Kleihues P, Bahr M, Ohgaki H, Ashkenazi A and Weller M (2001) Soluble decoy receptor 3 is 
expressed by malignant gliomas and suppresses CD95 ligand-induced apoptosis and chemotaxis. Cancer Res. 61: 2759-2765

19. Holler N, Tardivel A, Kovacsovics-Bankowski M, Hertig S, Gaide O, Martinon F, Tinel A, Deperthes D, Calderara S, Schulthess T, Engel J, Schneider P and Tschopp J (2003) Two adjacent trimeric Fas ligands are required for Fas signaling and formation of a death-inducing signaling complex. Mol. Cell Biol. 23: $1428-1440$

20. Ayroldi E, D'Adamio F, Zollo O, Agostini M, Moraca R, Cannarile L, Migliorati G, Delfino DV and Riccardi C (1999) Cloning and expression of a short Fas ligand: a new alternatively spliced product of the mouse Fas ligand gene. Blood 94: 3456-3467

21. Tanaka M, Itai T, Adachi M and Nagata S (1998) Downregulation of Fas ligand by shedding. Nat. Med. 4: 31-36

22. Schneider $\mathrm{P}$, Holler N, Bodmer JL, Hahne M, Frei K, Fontana A and Tschopp J (1998) Conversion of membrane-bound Fas(CD95) ligand to its soluble form is associated with downregulation of its proapoptotic activity and loss of liver toxicity. J. Exp. Med. 187: 1205-1213

23. Vargo-Gogola T, Crawford HC, Fingleton B and Matrisian LM (2002) Identification of novel matrix metalloproteinase-7 (matrilysin) cleavage sites in murine and human Fas ligand. Arch. Biochem. Biophys. 408: 155-161

24. Powell WC, Fingleton B, Wilson CL, Boothby M and Matrisian LM (1999) The metalloproteinase matrilysin proteolytically generates active soluble Fas ligand and potentiates epithelial cell apoptosis. Curr. Biol. 9: 1441-1447

25. Crawford HC, Scoggins CR, Washington MK, Matrisian LM and Leach SD (2002) Matrix metalloproteinase-7 is expressed by pancreatic cancer precursors and regulates acinar-to-ductal metaplasia in exocrine pancreas. J. Clin. Invest. 109: 1437-1444

26. Strand $S$, Vollmer $P$, van den Abeelen $L$, Kuball J, Heid $H$, Theobald M, Galle PR and Strand D (2002) Cleavage of CD95 (APO-1, Fas) by matrilysin (MMP7) induces apoptoaia resistance in tumor cells. Immunobiology 206: 247

27. Tanaka M, Suda T, Haze K, Nakamura N, Sato K, Kimura F, Motoyoshi K, Mizuki M, Tagawa S, Ohga S, Hatake K, Drummond AH and Nagata S (1996) Fas ligand in human serum. Nat. Med. 2: 317-322

28. Bahr GM, Capron A, Dewulf J, Nagata S, Tanaka M, Bourez JM and Mouton Y (1997) Elevated serum level of Fas ligand correlates with the asymptomatic stage of human immunodeficiency virus infection. Blood 90: 896-898

29. Aoki K, Kurooka M, Chen JJ, Petryniak J, Nabel EG and Nabel GJ (2001) Extracellular matrix interacts with soluble CD95L: retention and enhancement of cytotoxicity. Nat. Immunol. 2: 333-337

30. Seino K, Iwabuchi K, Kayagaki N, Miyata R, Nagaoka I, Matsuzawa A, Fukao K, Yagita H and Okumura K (1998) Chemotactic activity of soluble Fas ligand against phagocytes. J. Immunol. 161: 4484-4488

31. Ottonello L, Tortolina G, Amelotti M and Dallegri F (1999) Soluble Fas ligand is chemotactic for human neutrophilic polymorphonuclear leukocytes. J. Immunol. 162: 3601-3606

32. Igney FH and Krammer PH (2002) Immune escape of tumors: apoptosis resistance and tumor counterattack. J. Leukoc. Biol. 71: 907-920

33. Andreola G, Rivoltini L, Castelli C, Huber V, Perego P, Deho P, Squarcina $P$, Accornero P, Lozupone F, Lugini L, Stringaro A, Molinari A, Arancia G, Gentile M, Parmiani $G$ and Fais S (2002) Induction of lymphocyte apoptosis by tumor cell secretion of FasL-bearing microvesicles. J. Exp. Med. 195: 1303-1316

34. Rivoltini L, Carrabba M, Huber V, Castelli C, Novellino L, Dalerba P, Mortarini R, Arancia G, Anichini A, Fais S and Parmiani G (2002) Immunity to cancer: attack and escape in T lymphocyte-tumor cell interaction. Immunol. Rev. 188: 97-113

35. Hahne M, Rimoldi D, Schroter M, Romero P, Schreier M, French LE, Schneider P, Bornand T, Fontana A, Lienard D, Cerottini J and Tschopp J (1996) Melanoma cell expression of Fas(Apo-1/CD95) ligand: implications for tumor immune escape. Science 274: 1363-1366

36. Takahashi T, Tanaka M, Brannan Cl, Jenkins NA, Copeland NG, Suda T and Nagata S (1994) Generalized lymphoproliferative disease in mice, caused by a point mutation in the Fas ligand. Cell 76: 969-976

37. Rieux-Laucat F, Le Deist F and Fischer A (2003) Autoimmune lymphoproliferative syndromes: genetic defects of apoptosis pathways. Cell Death Differ. 10: 124-133

38. Viard I, Wehrli P, Bullani R, Schneider P, Holler N, Salomon D, Hunziker T, Saurat JH, Tschopp J and French LE (1998) Inhibition of toxic epidermal necrolysis by blockade of CD95 with human intravenous immunoglobulin. Science 282: 490-493
39. Grassme H, Kirschnek S, Riethmueller J, Riehle A, von Kurthy G, Lang F, Weller M and Gulbins E (2000) CD95/CD95 ligand interactions on epithelial cells in host defense to Pseudomonas aeruginosa. Science 290: 527-530

40. Raftery MJ, Schwab M, Eibert SM, Samstag Y, Walczak H and Schonrich G (2001) Targeting the function of mature dendritic cells by human cytomegalovirus: a multilayered viral defense strategy. Immunity 15: 997-1009

41. Matiba B, Mariani SM and Krammer PH (1997) The CD95 system and the death of a lymphocyte. Semin. Immunol. 9: 59-68

42. Kishimoto $\mathrm{H}$ and Sprent $\mathrm{J}(2000)$ The thymus and negative selection. Immunol. Res. 21: 315-323

43. Sprent $J$ and Kishimoto $H$ (2001) The thymus and central tolerance. Philos. Trans. R. Soc. Lond. B Biol. Sci. 356: 609-616

44. Singer GG and Abbas AK (1994) The fas antigen is involved in peripheral but not thymic deletion of $T$ lymphocytes in $T$ cell receptor transgenic mice. Immunity 1: 365-371

45. Janssen O, Stocker A, Sanzenbacher R, Oberg HH, Siddiqi MA and Kabelitz D (2000) Differential regulation of activation-induced cell death in individual human T cell clones. Int. Arch. Allergy Immunol. 121: 183-193

46. Janssen O, Sanzenbacher R and Kabelitz D (2000) Regulation of activationinduced cell death of mature T-lymphocyte populations. Cell Tissue Res. 301: 85-99

47. Blott EJ and Griffiths GM (2002) Secretory lysosomes. Nat. Rev. Mol. Cell Biol. 3: $122-131$

48. Stinchcombe JC, Bossi G, Booth S and Griffiths GM (2001) The immunological synapse of CTL contains a secretory domain and membrane bridges. Immunity 15: $751-761$

49. Suzuki I and Fink PJ (1998) Maximal proliferation of cytotoxic T lymphocytes requires reverse signaling through Fas ligand. J. Exp. Med. 187: $123-128$

50. Suzuki I and Fink PJ (2000) The dual functions of fas ligand in the regulation of peripheral CD8+ and CD4+ T cells. Proc. Natl. Acad. Sci. USA 97: 1707-1712

51. Desbarats J, Duke RC and Newell MK (1998) Newly discovered role for Fas ligand in the cell-cycle arrest of $\mathrm{CD} 4+\mathrm{T}$ cells. Nat. Med. 4: $1377-1382$

52. Suzuki I, Martin S, Boursalian TE, Beers C and Fink PJ (2000) Fas ligand costimulates the in vivo proliferation of CD8+ T cells. J. Immunol. 165: 55375543

53. Locksley RM, Killeen N and Lenardo MJ (2001) The TNF and TNF receptor superfamilies: integrating mammalian biology. Cell 104: 487-501

54. Wenzel J, Sanzenbacher R, Ghadimi M, Lewitzky M, Zhou Q, Kaplan DR, Kabelitz D, Feller SM and Janssen $O$ (2001) Multiple interactions of the cytosolic polyproline region of the CD95 ligand: hints for the reverse signal transduction capacity of a death factor. FEBS Lett. 509: 255-262

55. Scheu S, Alferink J, Potzel T, Barchet W, Kalinke U and Pfeffer K (2002) Targeted disruption of LIGHT causes defects in costimulatory T cell activation and reveals cooperation with lymphotoxin beta in mesenteric lymph node genesis. J. Exp. Med. 195: 1613-1624

56. Shaikh RB, Santee S, Granger SW, Butrovich K, Cheung T, Kronenberg M, Cheroutre $\mathrm{H}$ and Ware CF (2001) Constitutive expression of LIGHT on T cells leads to lymphocyte activation, inflammation, and tissue destruction. J. Immunol. 167: 6330-6337

57. Morel Y, Truneh A, Sweet RW, Olive D and Costello RT (2001) The TNF superfamily members LIGHT and CD154 (CD40 ligand) costimulate induction of dendritic cell maturation and elicit specific CTL activity. J. Immunol. 167: 2479-2486

58. Chou AH, Tsai HF, Lin LL, Hsieh SL, Hsu PI and Hsu PN (2001) Enhanced proliferation and increased IFN-gamma production in $\mathrm{T}$ cells by signal transduced through TNF-related apoptosis-inducing ligand. J. Immunol. 167: 1347-1352

59. Watts AD, Hunt NH, Wanigasekara Y, Bloomfield G, Wallach D, Roufogalis BD and Chaudhri G (1999) A casein kinase I motif present in the cytoplasmic domain of members of the tumour necrosis factor ligand family is implicated in 'reverse signalling'. EMBO J. 18: 2119-2126

60. Hane M, Lowin B, Peitsch M, Becker K and Tschopp J (1995) Interaction of peptides derived from the Fas ligand with the Fyn-SH3 domain. FEBS Lett. 373: 265-268

61. Ghadimi MP, Sanzenbacher R, Thiede B, Wenzel J, Jing Q, Plomann M, Borkhardt A, Kabelitz D and Janssen $O$ (2002) Identification of interaction 
partners of the cytosolic polyproline region of CD95 ligand (CD178). FEBS Lett. 519: $50-58$

62. Leo A and Schraven B (2001) Adapters in lymphocyte signalling. Curr. Opin Immunol. 13: 307-316

63. Lussier G and Larose L (1997) A casein kinase I activity is constitutively associated with Nck. J. Biol. Chem. 272: 2688-2694

64. Tanaka K (2000) Formin family proteins in cytoskeletal control. Biochem. Biophys. Res. Commun. 267: 479-481

65. Fuchs U, Rehkamp G, Haas OA, Slany R, Konig M, Bojesen S, Bohle RM, Damm-Welk C, Ludwig WD, Harbott J and Borkhardt A (2001) The human formin-binding protein 17 (FBP17) interacts with sorting nexin, SNX2, and is an MLL-fusion partner in acute myelogeneous leukemia. Proc. Natl. Acad. Sci. USA 98: 8756-8761

66. Worby CA and Dixon JE (2002) Sorting out the cellular functions of sorting nexins. Nat. Rev. Mol. Cell Biol. 3: 919-931
67. Blott EJ, Bossi G, Clark R, Zvelebil M and Griffiths GM (2001) Fas ligand is targeted to secretory lysosomes via a proline-rich domain in its cytoplasmic tail. J. Cell Sci. 114: 2405-2416

68. Gil D, Schamel WW, Montoya M, Sanchez-Madrid F and Alarcon B (2002) Recruitment of Nck by CD3 epsilon reveals a ligand-induced conformational change essential for T cell receptor signaling and synapse formation. Cell 109: $901-912$

69. Bossi G, Stinchcombe JC, Page LJ and Griffiths GM (2000) Sorting out the multiple roles of Fas ligand. Eur. J. Cell Biol. 79: $539-543$

70. Russell JH and Ley TJ (2002) Lymphocyte-mediated cytotoxicity. Annu. Rev. Immunol. 20: 323-370

71. Bossi G and Griffiths GM (1999) Degranulation plays an essential part in regulating cell surface expression of Fas ligand in T cells and natural killer cells. Nat. Med. 5: 90-96 